\title{
Minerais complexados na prevenção e tratamento de problemas locomotores em porcas
}

\author{
Complexed minerals on prevention and treatment \\ of locomotor problems in sows
}

Tatiana Carolina Gomes Dutra de Souza ${ }^{[a]}$,Emanuella Aparecida Pierozan ${ }^{[b]}$

[a] Médica Veterinária, Mestre em Ciências Veterinárias, Professora auxiliar do curso de Medicina Veterinária, Centro de Ensino Superior dos Campos Gerais (Cescage), Ponta Grossa, PR - Brasil, e-mail: tatianacdutra@hotmail.com

[b] Médica Veterinária, Mestre em Ciências Veterinárias, Universidade Federal do Paraná (UFPR), Curitiba, PR - Brasil, e-mail: emanuella@veterinaria.med.br

\section{Resumo}

O objetivo foi avaliar o efeito dos minerais complexados com metionina sobre a prevalência do grau de claudicação e sobre o tipo de lesões nos cascos traseiros de matrizes suínas, nulíparas e multíparas. Foram utilizadas 500 fêmeas comerciais Topigs, 100 nulíparas e 400 multíparas. As fêmeas foram alojadas em gaiolas individuais e no período experimental (01/06/2014 à 31/01/2015), a dieta foi suplementada com minerais complexados com metionina (50 ppm de Zn-metionina, 20 ppm Mn-metionina, 10 ppm de Cu-metionina). Os animais foram avaliados por inspeção visual quanto ao grau de claudicação e tipo de lesão nos cascos traseiros antes do fornecimento da dieta experimental e ao final da mesma. Os graus de severidade das lesões e claudicação foram avaliados dentro de cada grupo (nulíparas e multíparas), comparando a avaliação inicial com a avaliação final do experimento. A claudicação foi classificada conforme presença ou não da mesma, e quando presente foi identificada em: Grau 1, Grau 2, Grau 3 e Grau 4. As lesões foram classificadas em: Crescimento e Erosão da Almofada Plantar (AP), Rachadura Almofada Plantar-Sola (RAPS), Lesão de Linha Branca (LB), Rachadura Horizontal da Parede do casco (RHP), Rachadura Vertical da Parede do casco (RVP), Crescimento das Unhas (um) e Crescimento ou Ausência das Unhas Acessórias (UA). Os graus de severidade das lesões foram: 0 - normal; 1 - leve; 2 - moderada e 3 - grave. Para comparar os resultados obtidos com o oferecimento da nova dieta, utilizou-se o teste do qui-quadrado. Não houve diferença $(\mathrm{P}>0,05)$ quanto à claudicação nas fêmeas nulíparas em relação às multíparas nas duas avaliações realizadas. Porém, entre o período experimental, ocorreu redução $(\mathrm{P}<0,05)$ na prevalência de claudicação para os graus 1, 3 e 4 para as multíparas e para os graus 1, 2, 3 e 4 para as nulíparas. Na segunda avaliação, houve redução nas lesões de casco AP, RAPS, LB, RHP, RVP, um, UA nas fêmeas multíparas e nulíparas $(\mathrm{P}<0,05)$. A introdução de minerais complexados com metionina à dieta das fêmeas suínas reduziu todos os tipos das lesões de casco avaliados e a incidência dos diferentes graus de claudicação para fêmeas multíparas e nulíparas.

Palavras-chave: Cascos. Claudicação. Complexo metal-aminoácido. 


\section{Abstract}

The aim was to evaluate the effect of minerals complexed with methionine on the prevalence of lameness and on the type of lesions on hind hooves of sows, nulliparous and multiparous. Five hundred commercial females were used Topigs, 100 nulliparous and 400 multiparous. Females were housed in individual cages and the experimental period (from 06/01/2014 to 01/31/2015); the diet was supplemented with minerals complexed with methionine (50 ppm Zn-methionine, methionine 20 ppm Mn, 10 ppm Cu-methionine). The animals were evaluated by visual inspection as to the degree of lameness and type of injury in rear hooves before delivery of the diet and the end of it. The degrees of severity of lesions and lameness were evaluated in each group (nulliparous and multiparous) comparing the baseline to final evaluation of the experiment. Lameness was classified according to the presence or not of the same, and when this was identified in: Grade 1, Grade 2, Grade 3 and Grade 4. The lesions were classified into: Erosion and Growth of Paddy Planting (AP), Chap - Plantar Cushion Sole (RAPS), Injury White Line (LB), Horizontal Crack the wall (RHP), Vertical Crack the wall (PVR), Growth of nails (UN) and Absence of Growth and Nail Accessory (UA). The degree of severity of the injuries were adopted: 0 - normal; 1 - mild; 2 - moderate; 3 - severe. To compare the results obtained with the new diet offering, it was used the chi-square test. There was no difference $(P>0.05)$ on the lameness in nulliparous compared to multiparous in the two assessments performed. However, between the initial period and final evaluations, there was a reduction $(P<0.05)$ in the prevalence of lameness for grades 1,3 and 4 for the multiparous and for grades 1, 2, 3 and 4 for gilts. In the second evaluation, there was a reduction in hull damage AP, RAPS, LB, RHP, PVR, UN, UA in multiparous and nulliparous females $(P<0.05)$. The introduction of minerals complexed with methionine diet of sows reduced all kinds of assessed hull damage and the incidence of varying degrees of lameness for multiparous and nulliparous females.

Keywords: Hooves. Lameness. Amino acid complexes.

\section{Introdução}

Os prejuízos causados por claudicação e lesões de casco na produção brasileira de suínos podem estar subestimados por serem pouco estudados. Contudo, recentemente, alguns autores (Kramer et al., 2013; Souza e Kramer, 2013; Kramer e Alberton, 2014) observaram alta prevalência de doenças do sistema locomotor em matrizes no Brasil.

Problemas locomotores são indicadores de bem estar animal (Whay et al., 2003), pois o descarte precoce da matriz normalmente está vinculado às condições dolorosas (Deen, 2003). Além disso, as matrizes alojadas em confinamentos estão predispostas às lesões de casco. A seleção genética para alto desempenho, o tipo de piso, o espaçamento inadequado dos pisos ripados e a deficiência mineral são alguns dos fatores que facilitam o aparecimento de problemas locomotores em suínos (Anil et al., 2007; Alberton e Donin, 2013). Gjein e Larssen (1995) e Anil et al. (2007) observaram que entre 60 a $90 \%$ dessas matrizes possuem pelo menos um tipo de lesão que influencia no desempenho produtivo das mesmas.
Os problemas locomotores, de acordo com grau e tipo de lesão, ocasionam dor e dificultam o acesso ao alimento pela fêmea. Por consequência, a ingestão insuficiente de água contribui para desordens urinárias e o déficit energético influencia no desempenho reprodutivo da fêmea, destinando-as ao descarte precoce (Sobestiansky e Barcellos, 2007).

As lesões no casco acontecem por diversos fatores, entre eles genética, nutrição e manejo inadequados e má qualidade do piso. As matrizes que possuem rachaduras no casco, crescimento excessivo de unha, erosão e sobrecrescimento da almofada plantar apresentam performance reprodutiva prejudicada e maior incidência de claudicação (Vestergaard et al., 2006; Anil et al., 2008). As lesões que acometem o córion, como rachaduras profundas no casco e lesões de linha branca, são mais expressivas por ocasionarem reações inflamatórias, dor e claudicação. As lesões moderadas e severas colaboram de forma negativa para a longevidade da porca, intervindo nos índices zootécnicos da granja. 
Claudicação é um desvio do andar normal ocasionado por lesões, defeitos, doenças e/ou outros elementos que comprometam o aparelho locomotor. É um problema que ocorre devido às diversas desordens, como, por exemplo, osteocondrose e lesões de casco. Esse fator está relacionado com a perda da produtividade e descarte precoce das matrizes (Anil et al., 2008).

A manqueira afeta o desempenho reprodutivo da porca (Heinonen et al., 2006) e prejudica o desempenho da granja a longo prazo devido à retenção de reprodutoras com problemas de saúde no rebanho. As reprodutoras descartadas por causa da claudicação são removidas do rebanho com menor idade do que as fêmeas eliminadas por outras razões (Anil et al., 2009). É essencial que se proporcione o aumento da vida útil produtiva das porcas para reduzir os gastos com a reposição de marrãs.

Para melhorarmos o desempenho produtivo das porcas é fundamental amenizar a ocorrência de lesões no aparelho locomotor. A nutrição adequada exerce papel essencial para a qualidade dos cascos, com adequado balanço de nutrientes como proteína, minerais, vitaminas e energia (Tomlinson et al., 2004).

No Brasil, poucos estudos sobre a suplementação de minerais complexados que visam diminuir problemas locomotores em porcas são relatados. Porcas suplementadas com os microminerais complexados com aminoácidos apresentaram menos lesões e menor claudicação quando comparadas às fêmeas que receberam minerais inorgânicos na dieta (Anil et al., 2010). A suplementação de cobre, manganês e zinco complexados com aminoácidos reduzem a prevalência de lesões de cascos em fêmeas suínas (Anil, 2011). Dentro deste contexto, o objetivo deste trabaho foi avaliar o efeito dos minerais complexados com aminoácidos sobre a prevalência do grau de claudicação e sobre o tipo de lesões nos cascos traseiros de matrizes suínas, nulíparas e multíparas.

\section{Material e métodos}

0 experimento foi realizado no período do dia $01 / 06 / 2014$ ao dia $31 / 01 / 2015$, em uma granja comercial de suínos na região dos Campos Gerais (PR). Foram utilizadas 500 matrizes reprodutoras Topigs, 100 nulíparas e 400 fêmeas multíparas, que se encontravam nas fases de gestação e de maternidade, distribuídas do segundo ao quinto partos. As fêmeas foram alojadas em gaiolas individuais providas com comedouro e bebedouro individuais.

A análise dos cascos foi realizada duas vezes em cada fêmea, pelo mesmo avaliador, sendo uma avaliação ao início do experimento, em 01/06/2014, e a segunda avaliação ao final do experimento, em 31/01/2015.

Os cascos traseiros de cada fêmea foram avaliados conforme a presença e grau de severidade das lesões. A classificação adotada conforme o tipo de lesão observada pela inspeção visual foi: Crescimento e Erosão da Almofada Plantar (AP), Rachadura Almofada Plantar-Sola (RAPS), Lesão de Linha Branca (LB), Rachadura Horizontal da Parede do casco (RHP), Rachadura Vertical da Parede do casco (RVP), Crescimento das Unhas (um) e Crescimento ou Ausência das Unhas Acessórias (UA). De acordo com Deen et al. (2009), os graus de severidade das lesões adotados foram: 0 - normal; 1 - leve; 2 - moderada; 3 - grave.

As fêmeas foram avaliadas também em relação ao grau de claudicação, concomitantemente com a análise de casco. 0 exame clínico do aparelho locomotor foi realizado segundo a metodologia de Schulze et al. (1980). Quanto à claudicação, os animais foram movimentados no corredor entre as baias de gestação e maternidade e avaliaramse as matrizes por inspeção visual direta. As claudicações foram classificadas conforme ausência ou presença. Quando presente foi classificada quanto sua gravidade, sendo: Grau 1, alternância dos membros quando em estação e alterações discretas ao andar; Grau 2, alternância dos membros quando em estação e alterações perceptíveis ao andar; Grau 3 - alteração grave ao caminhar e matriz apoia com dificuldade o membro no chão, quando em estação; Grau 4, animal com dificuldade de se levantar, permanecendo deitado e, quando consegue se levantar, não apoia um dos membros no chão.

A dieta fornecida durante o período experimental estava de acordo com as exigências 
para a fase reprodutiva em que se encontrava cada fêmea, gestante ou lactante (Tabela 1).

Ambas as dietas foram formuladas de acordo com as recomendações das tabelas brasileiras (Rostagno, 2011), sendo suplementadas com minerais complexados com aminoácido (50 ppm de Zn-metionina, 20 ppm Mn-metionina, 10 ppm de Cu-metionina).

Cada fêmea consumiu ração lactação por 26 dias e, em seguida, 114 dias de ração gestação. Após esse período, cada matriz consumiu 26 dias de ração lactação seguidos por 79 dias de ração gestação, totalizando 245 dias de experimento (01/06/2014 à 31/01/2015). Não houve grupo controle para a dieta.

0 delineamento experimental foi inteiramente ao acaso, sendo utilizado o teste do qui-quadrado, ao nível de 5\% de significância, para comparar o uso de minerais complexados com metionina sobre a presença de claudicação e lesão de casco em dois grupos de matrizes suínas, nulíparas e multíparas. Ainda, os graus de severidade das lesões e de claudicação foram avaliados dentro de cada grupo (nulíparas e multíparas), comparando a avaliação inicial com a avaliação ao final do experimento. Para isso, utilizou-se o programa estatístico Minitab (McKenzie e Goldman, 1999).

\section{Resultados e discussão}

A prevalência de matrizes, multíparas e nulíparas, de acordo com o grau de claudicação para as duas avaliações, está relatada na Tabela 2 .

Não houve diferença quanto à presença de claudicação nas fêmeas nulíparas em relação às multíparas nas duas avaliações realizadas $(\mathrm{P}>0,05)$. Os resultados corroboram com Sobestiansky et al. (1989), que não constataram diferença na claudicação em matrizes nulíparas comparadas às multíparas. Contudo, ocorreu redução $(\mathrm{P}<0,05)$ na prevalência de lesões entre as avaliações inicial e final dentro do grupo das multíparas, para os graus 1, 3 e 4 de claudicação e, das nuliparas, para os graus, 1, 2, 3 e 4 de caludicação.

A prevalência do tipo de lesão das matrizes, multíparas e nulíparas, de acordo com o grau de lesão, para as duas avaliações, estão descritos na Tabela 3.

Houve menor $(\mathrm{P}<0,05)$ prevalência de cada tipo de lesão na segunda avaliação dentro do grupo das fêmeas multíparas e das nulíparas, em relação à 1a. avaliação. Ao total, $90 \%$ das fêmeas multíparas e $88 \%$ das fêmeas nulíparas apresentavam mais de um tipo de lesão, sendo que as lesões mais

Tabela 1 - Composição e valores analisados das dietas experimentais de gestação e lactação suplementadas com minerais ( $\mathrm{Zn}, \mathrm{Mn}, \mathrm{Cu}$ ) complexados com metionina

\begin{tabular}{lcc}
\hline & Tipo de dieta \\
\hline & Gestação & Lactação \\
\hline INGREDIENTES & & \\
\hline Milho 7,5\% (Kg) & 766 & 670 \\
Farelo de Soja 45\% (Kg) & 194 & 250 \\
Premix mineral/vitamínico (Kg) & 40 & 80 \\
Zn-metionina (ppm) & 50 & 50 \\
Mn-metionina (ppm) & 20 & 20 \\
Cu-metionina (ppm) & 10 & 10 \\
\hline VALORES ANALISADOS & & \\
\hline Proteína bruta (\%) & 17,79 & 18,62 \\
Cálcio (\%) & 0,99 & 0,80 \\
Fósforo total (\%) & 0,78 & 0,43 \\
Lisina (\%) & 1,51 & 0,85 \\
EM Suínos (Kcal/kg) & 3375,29 & 3480,00 \\
\hline
\end{tabular}


prevalentes foram Crescimento e Erosão da Almofada Plantar (AP) associadas à Rachadura Almofada Plantar-Sola (RAPS) para ambas as fêmeas, multíparas e nulíparas. Sobestiansky et al. (1989), Gjein e Larssen (1995), Souza e Kramer (2013) e Anil et al. (2007) também observaram maior prevalência de cascos com mais de um tipo de lesão em fêmeas suínas.

Neste estudo, os resultados corroboram com Anil et al. (2009), Anil et al. (2010), Anil et al. (2011) e Souza et al. (2013), que observaram redução de lesões nos cascos de fêmeas alojadas em gaiolas de gestação alimentadas com zinco, manganês e cobre complexados com metionina (50 ppm de $\mathrm{Zn}$, 20 ppm $\mathrm{Mn}, 10$ ppm de $\mathrm{Cu}$ ).

Ao avaliar vacas de leite, Siciliano-Jones et al. (2008) observaram que os minerais complexados com metionina contribuíram para o desempenho reprodutivo, a produção leiteira e a diminuição do grau de lesões de casco. A qualidade dos cascos bovinos melhorou com a suplementação de minerais complexados com metionina (Nocek et al., 2000; Nocek et al., 2006). As vacas de leite suplementadas com Zn-metionina apresentaram

Tabela 2 - Prevalência do grau de claudicação em matrizes suínas, multíparas e nulíparas, antes (1ª. avaliação) e após ( $2^{a}$. avaliação) alimentação com minerais complexados com metionina

\begin{tabular}{|c|c|c|c|c|c|}
\hline & \multicolumn{5}{|c|}{ Grau de claudicação } \\
\hline & Sem claudicação & Grau 1 & Grau 2 & Grau 3 & Grau 4 \\
\hline \multicolumn{6}{|c|}{ Fêmeas multíparas ${ }^{A}$} \\
\hline $1^{a}$. avaliação & $15,0 \mathrm{~b}$ & $21,0 \mathrm{a}$ & $30,0 \mathrm{a}$ & $25,0 \mathrm{a}$ & $09,0 \mathrm{a}$ \\
\hline $2^{a}$. avaliação & $26,0 \mathrm{a}$ & $18,0 \mathrm{~b}$ & $28,0 \mathrm{a}$ & $23,0 \mathrm{~b}$ & $05,0 \mathrm{~b}$ \\
\hline \multicolumn{6}{|c|}{ Fêmeas nulíparas A } \\
\hline $1^{\mathrm{a}}$. avaliação & $13,0 \mathrm{~b}$ & $34,0 \mathrm{a}$ & $24,0 \mathrm{a}$ & $22,0 \mathrm{a}$ & $07,0 \mathrm{a}$ \\
\hline $2^{a}$. avaliação & $27,0 \mathrm{a}$ & $30,0 \mathrm{~b}$ & $20,0 \mathrm{~b}$ & $17,0 \mathrm{~b}$ & $04,0 \mathrm{~b}$ \\
\hline
\end{tabular}

Legenda: A Diferença não significativa entre fêmeas multíparas e fêmeas nulíparas ( $P>0,05)$.

Nota: Letras minúsculas distintas na coluna diferem $(P<0,05)$ pelo teste de qui-quadrado

Tabela 3 - Prevalência do tipo de lesão em matrizes, multíparas e nulíparas, de acordo com o grau de lesão antes ( $1^{a}$. avaliação) e após ( $2^{a}$. avaliação) alimentação com minerais complexados com metionina

\begin{tabular}{|c|c|c|c|c|c|c|c|}
\hline & \multicolumn{7}{|c|}{ Tipo de lesão } \\
\hline & $A P^{1}$ & RAPS $^{2}$ & $\mathrm{LB}^{3}$ & $\mathrm{RHP}^{4}$ & $\mathrm{RVP}^{5}$ & $\mathrm{UN}^{6}$ & $U^{7}$ \\
\hline \multicolumn{8}{|c|}{ Fêmeas multíparas A } \\
\hline $1^{\mathrm{a}}$. avaliação & 93,8 a & $82,3 \mathrm{a}$ & 81,9 a & $50,6 a$ & $45,8 \mathrm{a}$ & 61,8 a & $79,7 a$ \\
\hline $2^{a}$. avaliação & $88,4 b$ & $42,3 b$ & $17,4 b$ & $23,6 b$ & $18,4 b$ & $58,6 b$ & $60,4 b$ \\
\hline \multicolumn{8}{|c|}{ Fêmeas nulíparas ${ }^{A}$} \\
\hline $1^{\text {a }}$. avaliação & 91,4 a & $79,6 a$ & $79,2 \mathrm{a}$ & $48,2 \mathrm{a}$ & $42,2 \mathrm{a}$ & $57,5 \mathrm{a}$ & $72,3 \mathrm{a}$ \\
\hline $2^{a}$. avaliação & $86,0 \mathrm{~b}$ & $37,9 b$ & $20,7 b$ & $20,1 b$ & $20,7 b$ & $48,2 b$ & $57,3 b$ \\
\hline
\end{tabular}

Legenda: Crescimento e Erosão da Almofada Plantar [AP], Rachadura Almofada Plantar-Sola [RAPS], Lesão de Linha Branca [LB], Rachadura Horizontal da Parede do casco [RHP], Rachadura Vertical da Parede do casco [RVP], Crescimento das Unhas [UN] e Crescimento ou Ausência das Unhas Acessórias [UA]. A Diferença não significativa entre Fêmeas multíparas e fêmeas nulíparas ( $P>0,05)$.

Nota: Letras minúsculas distintas na coluna diferem $(P<0,05)$ pelo teste de qui-quadrado. 
menor incidência de dermatite interdigital e rachaduras no talão (Spears, 1996).

Para frangos de corte provenientes de matrizes suplementadas com o complexo zinco-manganêsmetionina na dieta houve menor incidência de pododermatide, quando comparados aos frangos oriundos de matrizes que não receberam mineral complexado na dieta (Sanabria e Morales, 2008). Da mesma forma, Oliveira et al. (2010) concluíram que a adição de Zn-metionina contribui para a queratinização da pele e reduz o grau de pododermatites em frangos de corte.

\section{Conclusão}

A suplementação com 50 ppm de Zn-metionina, $20 \mathrm{ppm}$ Mn-Metionina e $10 \mathrm{ppm}$ de $\mathrm{Cu}$-metionina reduziu os graus de claudicação, respectivamente, para os graus 1, 2, 3 e 4, em 14,3\%, 6,7\%, 8,0\% e $44,4 \%$ nas fêmeas multíparas e 11,7\%, 16,6\%, $22,7 \%$ e $42,8 \%$ em fêmeas nulíparas.

As dietas com dosagens de 50 ppm de Znmetionina, $20 \mathrm{ppm}$ Mn-metionina, $10 \mathrm{ppm}$ de $\mathrm{Cu}$-metionina reduziram, respectivamente, em $5,75 \%, \quad 48,6 \%, \quad 78,7 \%, 53,35 \%, 59,8 \%, 5,1 \%$, 24,21\% o Crescimento e Erosão da Almofada Plantar, Rachadura Almofada Plantar-Sola, Lesão de Linha Branca, Rachadura Horizontal da Parede do casco, Rachadura Vertical da Parede do casco, Crescimento das Unhas e Crescimento ou Ausência das Unhas Acessórias nas fêmeas multíparas e, em fêmeas nulíparas, taxas de 5,9\%, 52,3\%, 73,8\%, $58,2 \%, 50,9 \% 16,1 \%, 20,74 \%$, respectivamente, para as mesmas lesões.

\section{Referências}

Alberton GC, Donin DG. Foco clínico diferenciado sobre os animais de reprodução relacionados com perdas reprodutivas. In: Simpósio Internacional de Produção Suína, 7., 2013, Foz do Iguaçu. Anais... Foz do Iguaçu: SUINTER; 2013

Anil SS, Anil L, Deen J, Baidoo SK, Walker RD. Factors associated with claw lesions in gestating sows. Journal Swine Health \& Production. 2007; 15(2):78-83.
Anil SS, Anil L, Deen J. Analysis of periparturient risk factors affecting sow longevity in breeding herds. Canadian Journal of Animal Science, 2008; 88(3):381389. doi:10.4141/CJAS07072.

Anil SS, Deen J, Anil L, Baidoo SK, Wilson ME, Ward TL. Evaluation of the supplementation of complexed trace minerals on the number of claw lesions in breeding sows. In: Manipulating Pig Production, 12., 2009, Cairns. Proceedings... Cairns: Australasian Pig Science Association. 2009; 108.

Anil SS, Deen J, Anil L, Baidoo SK, Wilson ME, Rapp C. Analysis of the effect of complexed trace minerals on the prevalence of lameness and severity of claw lesions in stall-housed sows. In: Joint Annual Meeting, 2010, Denver. Porceedings... Denver: JAM. 2010; 127.

Anil SS. Epidemiology of lameness in breeding female pigs. 2011. 126 f. Thesis (Doctorate in Philosophy), University of Minnesota, Minnesota, 2011.

Deen J. Sow longevity measurement. In: Allen Leman Swine Conference, 2003, Saint Paul. Proceedings... Saint Paul: Allen Leman Swine Conference. 2003; 192-193

Deen J, Schuttert M, Van Amstel S, Ossent P, Van Barneveld R. Feet First from Zinpro: Lesion Scoring Guide. Eden Prairie: Zinpro Corporation; 2009.

Gjein H, Larssen RB. The effect of claw lesions and claw infections on lameness in loose housing of pregnant sows. Acta Veterinaria Scandinavica. 1995; 36(4):451-459.

Heinonen M, Oravainen J, Orro T, Seppä-Lassila L, AlaKurikka E, Virolainen J, Tast A, Peltoniemi OA. Lameness and fertility of sows and gilts in randomly selected loose-housed herds in Finland. The Veterinary Record. 2006; 159(12):383-387.

Nocek JE, Johnson AB, Socha MT. Digital characteristics in commercial dairy herds fed metal-specific amino acid complexes. Journal of Dairy Science. 2000; 83(7):15531572. doi:10.3168/jds.S0022-0302(00)75028-4.

Nocek JE, Socha MT, Tomlinson DJ. The effect of trace mineral fortification level and source on performance of dairy cattle. Journal of Dairy Science. 2006; 89(7):2679-2693.

Kramer T, Souza TCGD, Teixeira AP, Alberton GC. Prevalência de lesões de casco em porcas da região Sul e Sudeste do Brasil. In: Associação Brasileira de Veterinários Especialistas em Suinocultura, 2013, Foz do Iguaçu. Anais... Foz do Iguaçu: ABRAVES, 2013. 
Kramer T, Alberton GC. Prevalência de claudicação de porcas e condições das gaiolas de gestação em granjas no Sul e Sudeste do Brasil. In: PorkExpo, 2014, Foz do Iguaçu. Anais... Foz do Iguaçu: PorkExpo, 2014.

McKenzie J, Goldman RN. The student edition of Minitab for windows manual: Release 12. Belmont: AddisonWesley; 1999.

Oliveira WNK, Silva JL, Lucena CAL, Silva KRL, Takata FN, Evêncio-Neto J. Uso do zinco orgânico na prevenção de lesões de pododermatite em aves. In: Jornada de Ensino, Pesquisa e Extensão, 10., 2010, Recife. Anais... Recife: JEPEX, 2010.

Rostagno HS. Tabelas brasileiras para aves e suínos: composição de alimentos e exigências nutricionais. 3. ed. Viçosa: UFV, 2011.

Sanabria RZ, Morales JA. Evaluación del efecto del complejo zinc-manganeso-aminoácido en pollo de engorde. Costa Rica: Centro de Investigaciones en Nutricion Animal (CINA) / Universidad de Costa Rica; 2008. (Informe Final - 29 f).

Schulze W, Bickhardt K, Bollwahn WV, Mickwitz G, Plonait H (Eds.). Klinik der Schweinekrankheiten. 1. ed. Hannover: Verlag M. \& H. Schaper; 1980.

Siciliano-Jones JL, Socha MT, Tomlinson DJ, DeFrain JM. Effect of trace mineral source on lactation performance, claw integrity, and fertility of dairy cattle. Journal of Dairy Science. 2008; 91(5):1985-1995. doi:10.3168/ jds.2007-0779.

Sobestiansky J, Wentz I, Silveira PRS, Munari J, Freitas AR. Ocorrência e caracterização das lesões nos cascos de fêmeas suínas reprodutoras. Revista da Faculdade de Medicina e Veterinária e Zootecnia da Universidade de São Paulo. 1989; 26(2):235-240. doi:10.11606/issn.23183659.v26i2p235-240.

Sobestiansky J, Barcellos DESN. Doenças dos suínos. 1. ed. Goiânia: Cânone Editorial, 2007.

Spears JW. Organic trace minerals in ruminant nutrition. Animal Feed Science and Technology. 1996; 58(1-2):151163. doi:10.1016/0377-8401(95)00881-0.

Souza TCGD, Kramer T. Minerais complexados com aminoácidos no tratamento e prevenção de lesões de casco em porcas. In: Associação Brasileira de Veterinários Especialistas em Suinocultura, 16., 2013, Foz do Iguaçu. Anais... Foz do Iguaçu: ABRAVES, 2013.
Tomlinson DJ, Mülling CH, Fakler TM. Invited Review: Formation of keratins in the bovine claw: roles of hormones, minerals, and vitamins in functional claw integrity. Journal of Dairy Science. 2004; 87(4):797-809. doi:10.3168/jds.S0022-0302(04)73223-3.

Vestergaard K, Bækbo P, Svensmark B. Sow mortality and causes for culling of sows in Danish pig herds. In: Congress of the International Pig Veterinary Society, 19., 2006, Copenhagen. Anais... Copenhagen: IPVS. 2006; 255 .

Whay HR, Main DCJ, Green LE, Webster AJF. Assessment of the welfare of dairy cattle using animal-based measurements: direct observations and investigation of farm records. Veterinary Record. 2003; 153(7):197-202. doi:10.1136/vr.153.7.197.

Recebido em: 17/05/2015

Received in: 05/17/2015

Aprovado em: 29/02/2016

Approved in: 02/29/2016 